\title{
Transfer reactions for nuclear astrophysics
}

\author{
Fairouz Hammache ${ }^{1, \star}$ \\ ${ }^{1}$ Institut de Physique Nucléaire d'Orsay, UMR8608, IN2P3-CNRS, Université Paris sud 11, 91406 Orsay, \\ France
}

\begin{abstract}
Direct measurements of cross sections at stellar energies are very challenging - if at all possible. This is essentially due to the very low cross-sections of the reactions of interest (especially when it involves charged particles), and/or to the radioactive nature of many key nuclei. Direct measurements with charged particles are often performed at higher energies and then extrapolated down to stellar energies using R-matrix calculations. However, these extrapolations are delicate because of the possible existence of unobserved low-energy or sub-threshold resonances. In order to bypass the difficulties related to direct measurements, indirect methods such as transfer reactions are used. These experiments are usually performed at higher energies and their conditions are relatively less stringent than in direct measurements. However, these methods rely on theoretical models for which the input parameters may be an important source of systematic uncertainties and thus need to be determined carefully. In this manuscript, a short overview on the difficulties related to direct measurements will be given as well as a description of the transfer reaction method and the theoretical concept behind. Finally, the method will be illustrated through two recent performed studies.
\end{abstract}

\section{Introduction}

Our understanding of stellar evolution in the Universe has been largely improved thanks to the interaction between three fields: observation, stellar modeling and nuclear physics. All these fields are in constant development: new telescopes and satellites open more and more windows on the cosmos, stellar modeling relies on ever-increasing computing and nuclear physics takes advantage of new facilities (radioactive beams high-intensity beams, underground laboratories) and sophisticated detection systems.

Nuclear reaction rates are one of the most important ingredients in describing how stars evolve. The study of the nuclear reactions involved in different astrophysical sites is thus mandatory to address most questions in nuclear astrophysics. Experimental techniques for cross sections determination lie in two main categories: direct measurements, in which the reaction of interest is reproduced, although the energy range may be different from the one in the stellar site; and indirect measurements, in which a different reaction is coupled with theoretical modeling to get the cross section of interest or to access the spectroscopic properties (Ex, $\mathrm{J}^{\pi}$, decay widths,...) of the involved nuclei.

Direct measurements at stellar energies are very challenging - if at all possible. This is essentially due to the very low cross-sections of the reactions of interest (especially when it involves charged

^e-mail: hammache@ipno.in2p3.fr 
particles), and/or to the radioactive nature of many key nuclei. Until recently, direct measurements with charged particles were often performed at higher energies and then extrapolated down to stellar energies using R-matrix calculations. However, these extrapolations are delicate because of the possible existence of unobserved low-energy or sub-threshold resonances. The effect of these resonances may change the extrapolated S-factor at astrophysical energies by a huge factor (sometimes orders of magnitude).

The other problem concerning the direct measurements is related to the radioactive nature of the nuclei involved in reactions occurring in explosive sites (novae, supernovae, X-ray bursts,...) and those involved in $(\mathrm{n}, \gamma)$ radiative captures in r-process and sometimes in s-process. The intensities of the radioactive beams are often low, rarely exceeding $10^{5}$ to $10^{6} \mathrm{pps}$ and for nuclei with relatively long half life, making targets with enough atoms per $\mathrm{cm}^{2}$ is very difficult. Hence the direct measurements of such reactions are very difficult and challenging and in case of r-process reactions it is currently impossible.

To bypass these difficulties (sub-threshold resonances, radioactive nuclei,...) indirect methods such as transfer reactions [1], Coulomb dissociation [2], ANC method [3] and Trojan Horse Method [4] are good alternatives. In these various methods, the experiments are usually performed at higher energies than the Coulomb barrier which imply higher cross sections than in direct measurements. Morevover these methods allow also to use stable beams to study reactions involving radioactive nuclei not far from the valley of stability. However, these methods are model dependent. They depend on the uncertainties relative to the different parameters used in the model. Hence, there are two sources of errors, experimental and theoretical. But the global uncertainty on the measured cross section can be reduced by combining different methods.

In this manuscript, I will focus on the the transfer reaction method. First of all I will show the possible type of reactions one can study using this method: the resonant one and the direct one. Then a description of the method and the theoretical concept behind will be given as well as some examples of experiments using this method.

\section{Resonant and direct reactions}

\subsection{Resonant reactions}

A resonant reaction, as shown in Fig. 1, is a two step process where the entrance channel $\mathrm{A}+\mathrm{x}$ forms first an excited state $\mathrm{E}_{x}$ in the compound nucleus $\mathrm{C}$ at the incident energy $\mathrm{E}_{c m}$ and then the formed resonant state decays either to the exit channel $\mathrm{B}+\mathrm{y}$ or to its ground state by emitting $\gamma$-rays. The resonant process can occur only at energies where the incident energy in center of mass is very close to the resonance energy which is given by: $\mathrm{E}_{r}=\mathrm{E}_{x}-\mathrm{Q}$ where $\mathrm{Q}$ is the $\mathrm{Q}$-value of the reaction.

The cross-section of a resonance is given by the Breit-Wigner formulae:

$$
\sigma(E)=\pi \lambda^{2} \frac{2 J_{C}+1}{\left(2 J_{A}+1\right)\left(2 J_{x}+1\right)} \frac{\Gamma_{x} \Gamma_{y}}{\left(E-E_{r}\right)^{2}+\left(\Gamma_{t o t} / 2\right)^{2}}
$$

And in the case of a narrow resonance $\left(\Gamma_{t o t} \ll \mathrm{E}_{r}\right)$, the reaction rate per particle pair is described by:

$$
<\sigma v>=\left(\frac{2 \pi}{\mu k T}\right)^{3 / 2}(\omega \gamma)_{R} \exp \left(-\frac{E_{r}}{k T}\right)
$$

where the resonance strength is given by:

$$
(\omega \gamma)_{R}=\frac{2 J_{C}+1}{\left(2 J_{A}+1\right)\left(2 J_{x}+1\right)} \frac{\Gamma_{x} \Gamma_{y}}{\Gamma_{t o t}}
$$




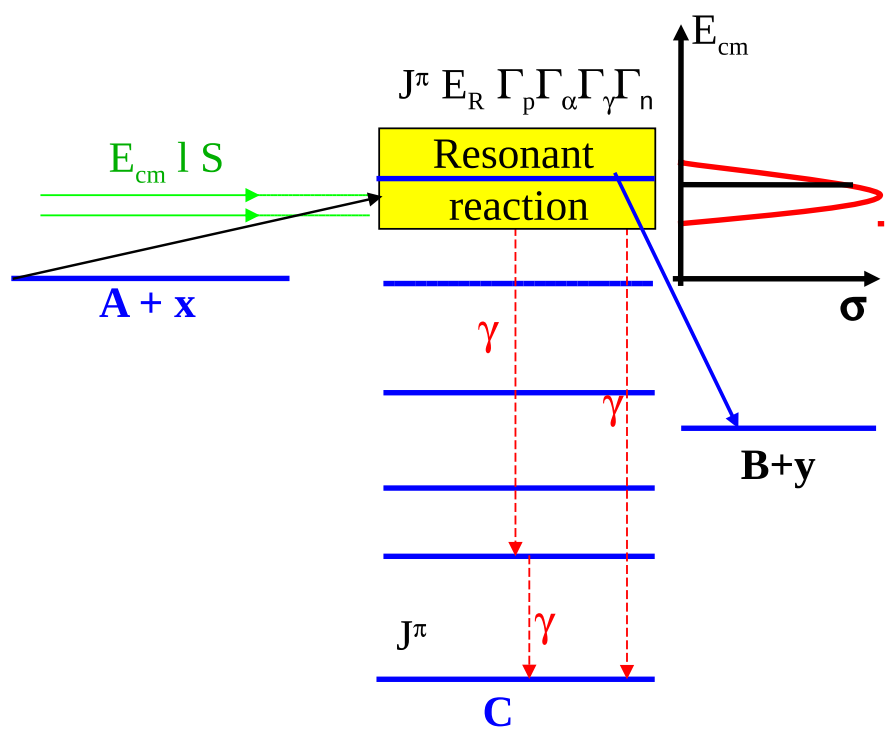

Figure 1. Resonant reaction scheme

The important parameters to describe a resonant cross-section and calculate the reaction rate are the resonance energy $\mathrm{E}_{r}$, the partial width $\Gamma_{x}$ which expresses the probability to form the compound nucleus $C^{*}$ in the excited state $\mathrm{E}_{x}$, the partial width $\Gamma_{y}$ which expresses the probability of decay to the exit channel $\mathrm{B}+\mathrm{y}$ or $\mathrm{C}+\gamma$ and the spin-parity of the states involved in the compound nucleus $\mathrm{J}_{C}$.

Once these different parameters are known, one can easily calculate the reaction rate using formulae (1). The excitation energy and the partial decay widths of the states of interest can be obtained from transfer reactions experiments.

Let's assume a compound nucleus $\mathrm{C}$ is in an excited state $\mathrm{E}_{r}$ (see Fig. 1) with a pure core-particle configuration $|A \otimes x\rangle$. Thanks to the time reverse invariance of the electro-magnetic and nuclear processes, the partial width of the formation of the compound nucleus in the excited state $\mathrm{E}_{r}$ through $\mathrm{A}+\mathrm{x}$ is identical to the partial width of the decay of the excited state $\mathrm{C}^{*}$ into $\mathrm{A}+\mathrm{x}$. The decay partial width of $\mathrm{C}$ into $\mathrm{A}+\mathrm{x}$ is given by the following formulae:

$$
\Gamma_{x}^{s . p}=\frac{\hbar^{2}}{\mu} R P_{l}(E, R)|\varphi(R)|^{2}
$$

with

$$
\left.\int_{0}^{\infty} \varphi(R)\right|^{2} R^{2} d R=1
$$

Where $\mathrm{R}$ is is the channel radius, $\mathrm{P}_{l}$ is the penetrability factor, $\mathrm{E}$ is the energy of the free particle $\mathrm{x}$ and $\varphi(\mathrm{R})$ is the radial wave function of the particle $\mathrm{x}$. Hence, for a state with a pure core particle 
configuration, the single particle $\Gamma_{x}^{s . p}$ can be calculated. But in most cases, the states are not pure core-particle configurations, they are a mixture of configurations which decay or formation partial width $\Gamma_{x}$ is given by the product of the single particle width $\Gamma_{x}^{s p}$ and the spectroscopic factor $\mathrm{S}$ :

$$
\Gamma_{x}=\Gamma_{x}^{s p} \times C^{2} S
$$

where $\mathrm{C}$ is the isospin Clebsch-Grodon coefficient.

The spectroscopic factor $\mathrm{S}$ expresses the overlap probability between the antisymmetrized wave functions of the entrance channel $\mathrm{A}+\mathrm{x}$ and the final state $\mathrm{C} ; \mathrm{S}=\langle A+x \mid C\rangle$. Hence, by determining the spectroscopic factor $\mathrm{S}$ via transfer reactions, one can then calculate $\Gamma_{x}$ or the reduced width as we will see in section 3 .

\subsection{Direct $(n, \gamma)$ captures}

For reactions where direct capture mechanism can sometime plays an important role such as in ${ }^{48} \mathrm{Ca}(\mathrm{n}, \gamma){ }^{49} \mathrm{Ca}$ reaction [5], the capture occurs on bound states of the final nucleus in a one step process (see Fig. 2). The captures are possible at all neutron energies and the cross section varies smoothly with the neutron energy.

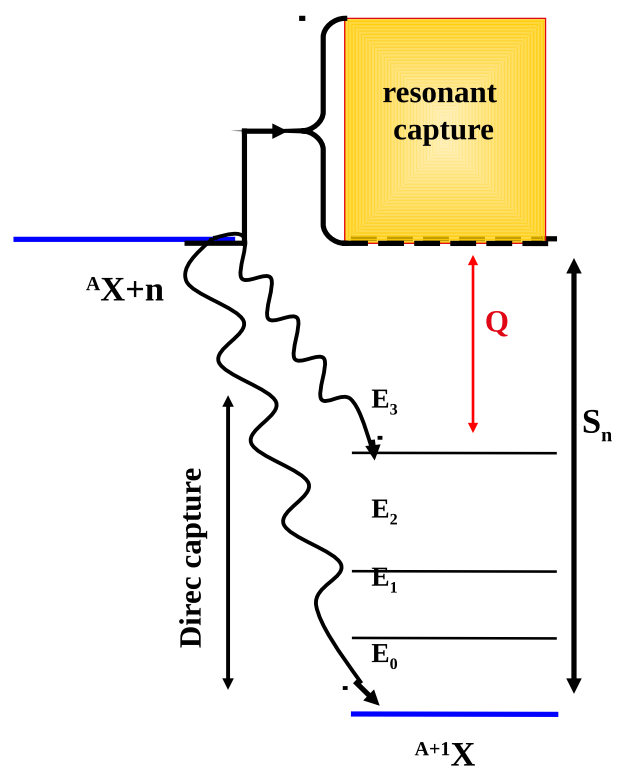

Figure 2. Direct capture reaction scheme

The cross section of the direct process is described by the following single matrix element [5]:

$$
\sigma_{(n, \gamma)}=\sum_{i} C_{i}^{2} S_{i}\left|\int_{r=0}^{\infty} \phi_{f} \theta_{e m} \phi_{i} d \vec{r}\right|^{2}
$$


Where $\theta_{e m}$ is the electro-magnetic multipole transition operator, $\phi_{i}$ and $\phi_{f}$ are the scattering and the bound state wave functions in the entrance and exit channels, respectively. The wave functions are Schrödinger's equation solutions with the potential $\mathrm{V}$ obtained by double folding and $\mathrm{S}$ is the spectroscopic factor of the final state. The double folding potential $\mathrm{V}$ is given by:

$$
V=\lambda \iint \rho_{n}\left(\vec{r}_{n}\right) \rho_{A}\left(\vec{r}_{A}\right) v_{e f f}\left(E_{n}, \rho_{n}, \rho_{A},|\vec{R}-\vec{r}|\right) d \vec{r}_{n} d \vec{r}_{A}
$$

where $\mathrm{v}_{\text {eff }}$ is the nucleon-nucleon interaction, $\rho_{n}$ is the neutron density, $\rho_{A}$ is the nucleus target density and $\lambda$ is a factor adjusted to reproduce the elastic scattering data for the entrance channel and the neutron separation energy for the exit channel. The latter depends on the location of the excited states in the final nucleus. The spectroscopic information on the low energy bound states $\left(\mathrm{E}_{x}, 1, \mathrm{C}^{2} \mathrm{~S}\right)$ are accessible via $(\mathrm{d}, \mathrm{p})$ transfer reactions in case of $(\mathrm{n}, \gamma)$ reaction.

\section{Transfer Reaction Method}

Let's assume that we want to study a resonant reaction $\mathrm{x}+\mathrm{A} \rightarrow \mathrm{C}^{*} \rightarrow \mathrm{B}+\mathrm{y}$ and measure the partial decay width $\Gamma_{x}$ of the state of interest in $C^{*}$ into the entrance channel $\mathrm{A}+\mathrm{x}$. To do so, we populate the excited states of $\mathrm{C}$ by transfering the small particle $\mathrm{x}$ (see Fig. 3) which can be a nucleon or a cluster of nucleons from the nucleus a to the nucleus A.

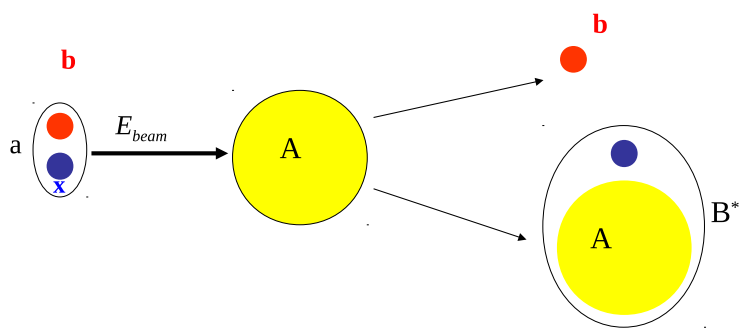

Figure 3. Sketch of a transfer reaction before (left) and after reaction (right).

This will feed the valence states of the final nucleus $\mathrm{C}$, hopefully with no perturbation of the core, that is why it is called one step direct transfer reaction. The other part of the projectile $\mathrm{b}$ will continue its movement and will be detected. By measuring the emitted angle and energy of the particle $\mathrm{b}$, one can deduce the energy of the excited state that was populated in $\mathrm{C}$ from kinematics and by comparing the shape of the measured angular distributions to those predicted by the distorted wave Born approximation theory (DWBA), one can extract the orbital angular momentum 1 of the populated single particle bound state. The $\mathrm{j}$-values can also be obtained from polarization measurements.

The theoretical direct transfer cross section is calculated using the DWBA formalism and it is given by the following matrix element:

$$
\left(\frac{d \sigma}{d \Omega}\right)_{D W B A} \propto\left|<\chi_{f} I_{x A}^{C}\left(r_{x A}\right)\right| V\left|I_{b x}^{X}\left(r_{b x}\right) \chi_{i}>\right|^{2}
$$


Where $\chi_{i, f}$ are the distorted wave functions of the initial and the final state, $\mathrm{V}$ is the transition transfer operator, $\mathrm{I}_{x A}{ }_{x A}\left(\mathrm{r}_{x A}\right)$ is the overlap function of the final bound state $\mathrm{C}$ formed by $\mathrm{A}+\mathrm{x}$ and $\mathrm{I}^{X}{ }_{b x}\left(\mathrm{r}_{b x}\right)$ is the overlap function of the bound state a formed by $\mathrm{b}+\mathrm{x}$.

The radial part of these last functions is given by the following product:

$$
I_{\beta \gamma}^{\alpha}\left(r_{\beta \gamma}\right)=S^{1 / 2} \varphi_{\beta \gamma}\left(r_{\beta \gamma}\right)
$$

Where $\mathrm{S}$ is the spectroscopic factor, $\varphi_{\beta \gamma}(\mathrm{r})$ is the radial wave function of the bound state $\mathrm{C}$ or a with $\alpha$ being the final bound state $\mathrm{C}$ or the bound state a, $\beta$ being the transfered particle $\mathrm{x}$ and $\gamma$ being A or b respectively.

The spectroscopic factor of the different populated states in $\mathrm{C}$ can be extracted from the ratio of the measured differential cross section to the one calculated by DWBA:

$$
\left(\frac{d \sigma}{d \Omega}\right)_{\exp }=S_{1} S_{2}\left(\frac{d \sigma}{d \Omega}\right)_{D W}
$$

As one can see in formulae 11, there are two spectroscopic factors, $S_{1}$ for the final bound state of interest in the exit channel and $S_{2}$ for the bound state in the entrance channel. Hence, the knowledge of the spectroscopic factor $S_{2}$ is mandatory to extract the the spectroscopic factor of interest $S_{1}$. Once the spectroscopic factor of the state of interest is extracted, one can then determine the reduced decay width using the following formula [6]:

$$
\gamma_{x}^{2}=\frac{\hbar^{2} R}{2 \mu} S_{x}|\varphi(R)|^{2}
$$

where $\varphi(\mathrm{R})$ is the radial wave function of the bound state $\mathrm{C}$ formed by $\mathrm{A}+\mathrm{x}$, calculated at a channel radius $\mathrm{R}$ where $\varphi(\mathrm{R})$ has its asymptotic behavior. The calculation of $\Gamma_{x}$ is then given by:

$$
\Gamma_{x}=2 P_{l} \gamma_{x}^{2}
$$

where $\mathrm{P}_{l}$ is the penetrability.

One can also extract the ANC [3] using the expression:

$$
\tilde{C}^{2}=S_{\alpha} \frac{R^{2} \varphi^{2}(R)}{\tilde{W}^{2}(R)}
$$

where $\mathrm{W}(\mathrm{R})$ is the Whittaker function.

To calculate the transfer DWBA differential cross sections, a number of computer codes can be used such as FRESCO [7] and DWUCK [8]. In these codes, a number of inputs are needed and they have to be carefully determined. These inputs are the optical potential parameters describing the entrance channel, those describing the exit channel and finally the potential well parameters describing the interaction of the transfered particle with the core in the final and initial nucleus. For the optical potential parameters of the entrance and exit channel, elastic scattering measurements are needed. When elastic measurements are not available, one can use the potentials from measurements performed in the mass region close to the nuclei of interest and at close incident energies or the global potential formulae from Perey and Perey [9] abd Daehnick et al. [10]. Concerning the well parameters of the Woods-Saxon potential, the depth is adjusted to reproduce the binding energy of the bound state. For the radius and the diffuseness, different realistic sets can be used and the selected ones are those giving the optimal fit of the measured angular distributions. Note that the accuracy on the 
extraction of the spectroscopic factor can hardly be better than $20 \%$, so this method can not be used for reactions where an uncertainty better than $10 \%$ is needed.

The calculation of the transfer reaction cross sections with DWBA formalism involves the evaluation of a six dimensional integral [1]. For light-ion reactions such as $(\mathrm{d}, \mathrm{p})$ reaction, the direct reaction causing the transition is often approximated by a zero-range potential. This is partially justified by the small size of the light nucleus (e.g:deuteron) in comparison with the size of the other nuclei and the S wave state of the projectile. With this assumption, the DWBA integrals reduce to three-dimensional integrals that make the calculation simpler as only the form factor describing the interaction of the transfered particle with the core in the final nucleus has to be considered. However, this zero range assumption is no more valid if the projectile is not in an $S$ wave internal state $(\mathrm{L}=0)$ and in case of reactions where the transferred particle is a cluster of more than 2 nucleons such as the $\alpha$-particle. Here, the relatively large size of the nucleus $\left({ }^{6} \mathrm{Li}\right.$ or ${ }^{7} \mathrm{Li}$ in case of $\alpha$ transfer) from which the cluster is transferred, together with the small wavelength associated with the relative motion, makes this assumption very poor and sometimes wrong. For these cases, finite range DWBA calculations have to be performed with a six dimensional integral which includes both form factors describing the interaction of the transferred particle with the core in the initial nucleus and in the final nucleus respectively.

When using the transfer method, one has to be aware that other reaction mechanisms such as the compound nucleus mechanism, the multi-step transfer reaction mechanism, the projectile breakup and the transfer to continuum [1] can occur. The contribution of these mechanisms can be evaluated by using Hauser Feschbach calculations, coupled channel calculations and continuum discretized coupled channel (CDCC) calculations respectively [7]. Note that usually the direct transfer mechanism is favored at small centre-of-mass angles $\left(\theta_{c . m} \leqslant 50^{\circ}\right)$, so one has to perform the transfer experiments at detection angles and incident energies where the compound nucleus component is reduced.

\subsection{Examples of transfer reactions}

Various type of transfer reactions can be used to study different reactions of astrophysical interest. The $\left({ }^{3} \mathrm{He}, \mathrm{d}\right)$ and $(\mathrm{d}, \mathrm{n})$ proton transfer reactions are used to evaluate $(\mathrm{p}, \gamma)$ reactions by extracting the excitation energy, momentum and the proton spectroscopic factors of the states of interest as in Parikh et al.'s experiment [11]. The (p,d) and $(\mathrm{p}, \mathrm{t})$ transfer reactions are used to study proton-rich nuclei involved in reactions of astrophysical interest $[12,13]$. For both of these reactions, the Q-values are strongly negative, hence proton beam with energies larger than $30 \mathrm{MeV}$ are needed. Another interest of using $(\mathrm{p}, \mathrm{t})$ reactions comes from its selectivity, it populates only natural spin and parity states of even-even nuclei. To evaluate direct $(\mathrm{n}, \gamma)$ reaction cross-sections, one can use $(\mathrm{d}, \mathrm{p})$ neutron transfer reactions. In this case, one needs to use Adiabatic distorted wave approximation (ADWA) calculation which takes into account the deuteron breakup for deuterons energies larger than $20 \mathrm{MeV}$ [14]. The $\alpha$ transfer reactions are very useful to study the properties of nuclei involved in $\alpha$-induced reactions such as $(\alpha, \gamma)$ and $(\alpha, \mathrm{n})$ of astrophysical interest. The most commonly used are $\left({ }^{6} \mathrm{Li}, \mathrm{d}\right)$ and $\left({ }^{7} \mathrm{Li}, \mathrm{t}\right)$ reactions. From the Finite-range DWBA analysis of the measured angular distributions, one can extract the $\alpha$ spectroscopic factors and thus the ANCs and/or the $\alpha$ reduced widths of the states of interest needed to calculate the $(\alpha, \mathrm{n})$ and $(\alpha, \gamma)$ cross sections. According to previous works $[6,15,16]$, the use of ${ }^{7} \mathrm{Li}$ instead of ${ }^{6} \mathrm{Li}$ reduces possible multi-step effects and the transfer cross sections to low spin states are enhanced because of the non-zero $\alpha$ angular momentum in ${ }^{7} \mathrm{Li}$ as observed when comparing the transfer reactions $\left({ }^{6} \mathrm{Li}, \mathrm{d}\right)$ and $\left({ }^{7} \mathrm{Li}, \mathrm{t}\right)$ on ${ }^{12} \mathrm{C}$. These transfer reactions were applied to many astrophysical studies such as ${ }^{12} \mathrm{C}(\alpha, \gamma){ }^{16} \mathrm{O}$ [17], ${ }^{13} \mathrm{C}(\alpha, \mathrm{n}){ }^{16} \mathrm{O}$ [18] and ${ }^{22} \mathrm{Ne}(\alpha, \mathrm{n}){ }^{25} \mathrm{Mg}$ [19].

An illustration of the transfer method will be given in the following through two examples: first the study of the resonant reaction ${ }^{13} \mathrm{C}(\alpha, \mathrm{n}){ }^{16} \mathrm{O}$ via a $\left({ }^{7} \mathrm{Li}, \mathrm{t}\right) \alpha$-transfer reaction and the study of the direct component of ${ }^{60} \mathrm{Fe}(\mathrm{n}, \gamma){ }^{61} \mathrm{Fe}$ reaction via ${ }^{60} \mathrm{Fe}(\mathrm{d}, \mathrm{p}){ }^{61} \mathrm{Fe}$ transfer reaction in inverse kinematics. 


\subsubsection{Application of the method to a resonant case: ${ }^{13} \mathrm{C}(\alpha, n)^{16} \mathrm{O}$}

Nearly half of the heavy elements observed in the Universe are produced by a slow sequence of neutron capture reactions, the so-called s-process. For this astrophysical process, two neutron sources are used in all evolution models: the reactions ${ }^{13} \mathrm{C}(\alpha, \mathrm{n}){ }^{16} \mathrm{O}$ and ${ }^{22} \mathrm{Ne}(\alpha, \mathrm{n}){ }^{25} \mathrm{Mg}$. For the AGB stars of 1-3 solar masses at low temperatures, only the ${ }^{13} \mathrm{C}(\alpha, \mathrm{n}){ }^{16} \mathrm{O}$ reaction is considered as the main neutron source for the s-process [20]. Hence, all the models describing the s-process nucleosynthesis in these AGB stars depend on the neutron flux from the ${ }^{13} \mathrm{C}(\alpha, \mathrm{n}){ }^{16} \mathrm{O}$ reaction and so on the cross section of this reaction which occurs in these stars at temperatures around $10^{8} \mathrm{~K}$, i.e around the Gamow peak $\mathrm{E}_{c m} \sim 190 \mathrm{keV}$.

Direct measurement of ${ }^{13} \mathrm{C}(\alpha, \mathrm{n}){ }^{16} \mathrm{O}$ reaction at this energy is extremely difficult because the cross section decreases drastically when the incident $\alpha$ energy decreases. Thus, direct measurements [21] have only been performed down to $260 \mathrm{keV}$ too far away from the energy range of interest around $190 \mathrm{keV}$. R-matrix extrapolations [22] of the cross sections measured at higher energies have then to be performed, including the contribution of the $6.356 \mathrm{MeV}, 1 / 2^{+}$, state of the compound nucleus ${ }^{17} \mathrm{O}$, which lies $3 \mathrm{keV}$ below the $\alpha+{ }^{13} \mathrm{C}$ threshold. The contribution of this sub-threshold state strongly depends on its $\alpha$-spectroscopic factor, $\mathrm{S}_{\alpha}$. R-matrix extrapolations which consider a large value of 0.7 for the $\alpha$ spectroscopic factor $\left(\mathrm{S}_{\alpha}\right)$ of the subthreshold state at $6.356 \mathrm{MeV}$ lead to a rapid increase of the astrophysical $\mathrm{S}$-factor when the energy decreases to zero while they lead to constant extrapolated $\mathrm{S}$-factor at low energies when they use a null $\mathrm{S}_{\alpha}$.

Investigation of the effect of the sub-threshold resonance on the astrophysical S-factor was performed through a determination of the alpha spectroscopic factor of the $6.356 \mathrm{MeV}$ state using the $\alpha$-transfer reactions ${ }^{12} \mathrm{C}\left({ }^{6} \mathrm{Li}, \mathrm{d}\right){ }^{17} \mathrm{O}[23,24]$ and ${ }^{13} \mathrm{C}\left({ }^{7} \mathrm{Li}, \mathrm{t}\right){ }^{17} \mathrm{O}$ [18]. The experiment [18] was performed using a ${ }^{7} \mathrm{Li}^{3+}$ beam of 34 and $28 \mathrm{MeV}$ energy provided by the Orsay TANDEM impinging on a self-supporting enriched ${ }^{13} \mathrm{C}$ target. The reaction products were analyzed with an Enge Split-pole magnetic spectrometer (see Fig. 4) and the tritons were detected at various angles in the laboratory system.

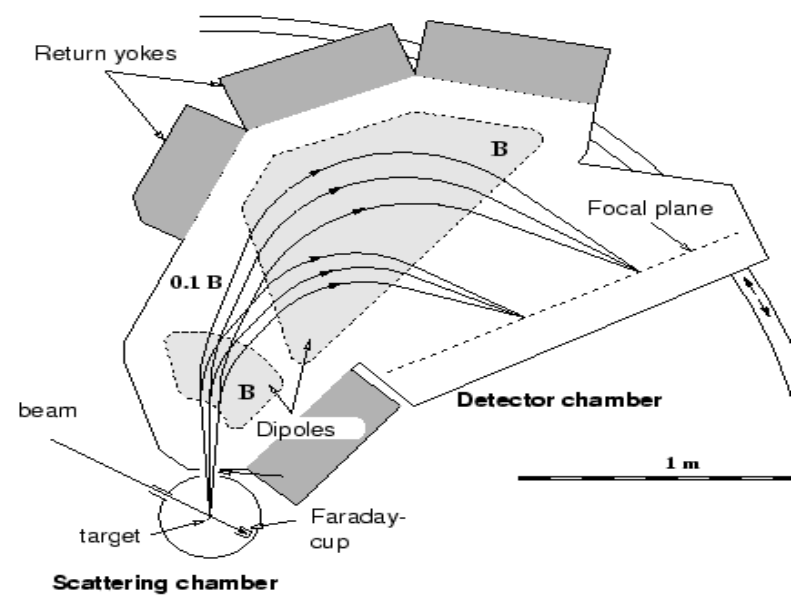

Figure 4. Schematic view of the Split-Pole spectrometer.

The measurements were performed at two incident energies in order to check the direct character of the transfer mechanism. Elastic scattering measurements were also performed at $28 \mathrm{MeV}$ incident 
energy in order to get realistic optical potential parameters for the entrance channel. The measured elastic scattering angular distribution is displayed in Fig. 5.

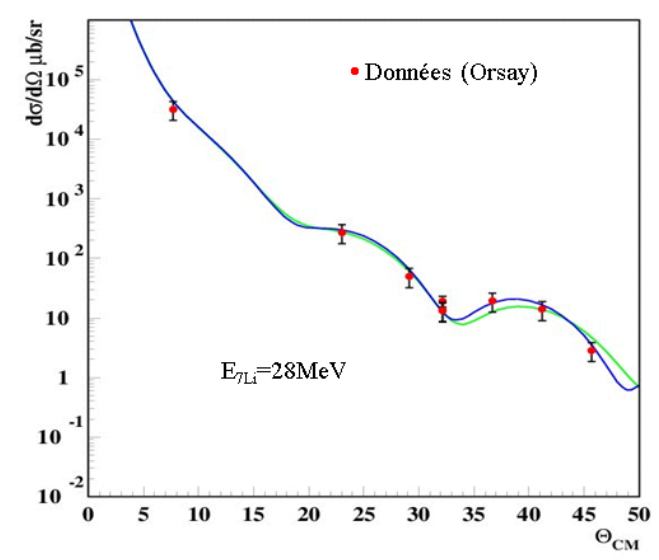

Figure 5. Experimental elastic ${ }^{13} \mathrm{C}\left({ }^{7} \mathrm{Li}^{7}\right)^{13} \mathrm{C}$ differential cross sections obtained at $28 \mathrm{MeV}$ compared with DWBA calculations.

The experimental ${ }^{13} \mathrm{C}\left({ }^{7} \mathrm{Li}, \mathrm{t}\right){ }^{17} \mathrm{O}$ differential cross sections measured for the 6.356, 3.055, 4.55 and $7.38 \mathrm{MeV}$, at the two incident energies of 34 and $28 \mathrm{MeV}$, are displayed in Fig. 6 together with the Finite-range DWBA calculations, using the FRESCO code [7]. See [18] for details.

Many combinations of entrance and exit optical potentials parameters were investigated. Concerning the entrance channel, several ${ }^{7} \mathrm{Li}+{ }^{13} \mathrm{C}$ optical potentials extracted from previous elastic measurements [25] at $34 \mathrm{MeV}$ and the present elastic measurements at $28 \mathrm{MeV}$ were tested. For the exit triton channel, optical potential parameters from Garrett et al. [26] were selected. The dependence of the calculation to the ${ }^{13} \mathrm{C}-\alpha$ interaction potential was also investigated. A spreading of about $30 \%$ on $\mathrm{S}_{\alpha}$ was found when the optical potential parameters were varied and another $20 \%$ with the variation of the well parameters. Finally, the selected optical and interaction parameters are those giving the best fit, using the maximum likelihood function set at $3 \sigma$ level, for the studied states $(6.05,6.13,6.92$, $7.12 \mathrm{MeV}$ ) at both incident energies.

The $\alpha$-spectroscopic factors were extracted from the normalization of the finite-range DWBA curves to the experimental data. The good agreement between the DWBA calculations and the measured differential cross sections of the different excited states of ${ }^{17} \mathrm{O}$ at the two bombarding energies of $28 \mathrm{MeV}$ and $34 \mathrm{MeV}$ respectively, gives strong evidence of the direct nature of the $\left({ }^{7} \mathrm{Li}, \mathrm{t}\right)$ reaction populating these levels and confidence in the DWBA calculations. An $\mathrm{S}_{\alpha}$ mean value of $0.29 \pm 0.11$ is deduced for the sub-threshold state at $6.356 \mathrm{MeV}$ in ${ }^{17} \mathrm{O}$. The uncertainty on the extracted $\alpha$ spectroscopic factor for the state of interest $6.356 \mathrm{MeV}$ was evaluated from the dispersion of the deduced $\mathrm{S}_{\alpha}$ values at the two incident energies and using different sets of optical potentials in the entrance and exit channels and different $\alpha-{ }^{13} \mathrm{C}$ well geometry parameters [18].

The $\alpha$-reduced width $\gamma_{\alpha}^{2}$ of about $13.5 \pm 6.6 \mathrm{keV}$ for the $6.356 \mathrm{MeV}$ state was obtained using Eq.12. The calculation was performed at the radius $\mathrm{R}=7.5 \mathrm{fm}$ where the Coulomb asymptotic behavior of the radial part of the $\alpha-{ }^{13} \mathrm{C}$ wave function is reached. 

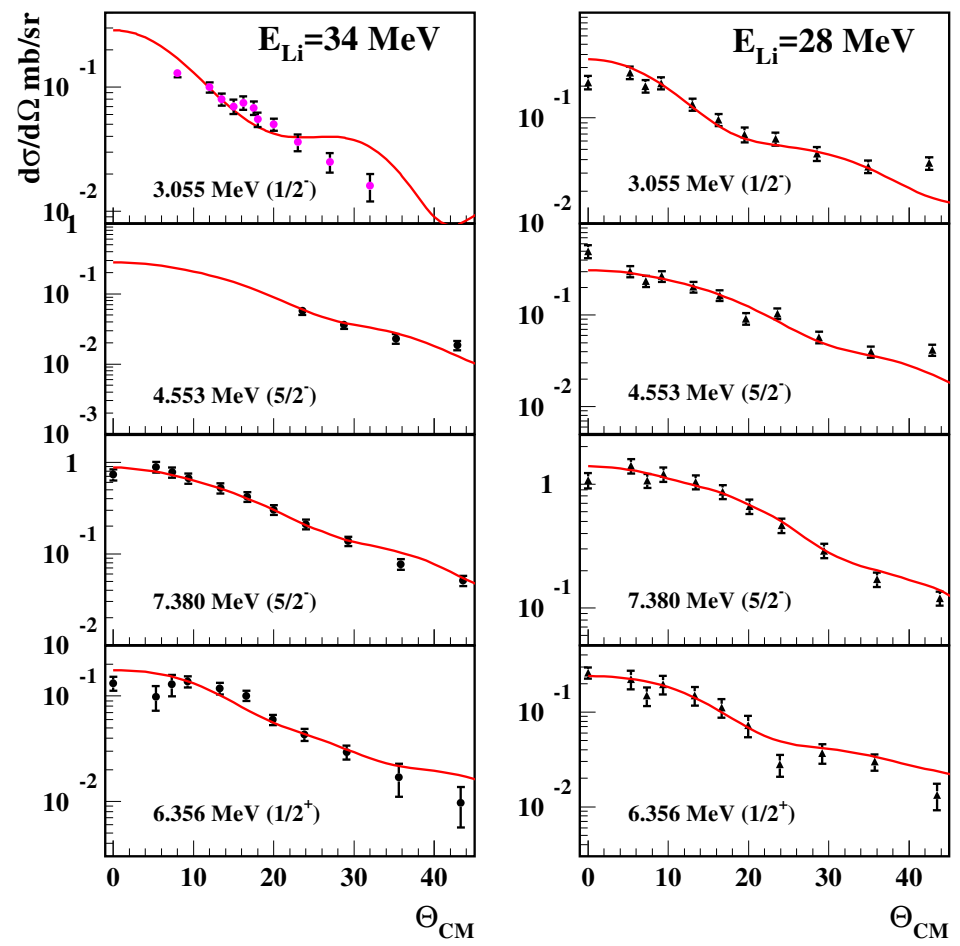

Figure 6. Experimental differential cross sections of the ${ }^{13} \mathrm{C}\left({ }^{7} \mathrm{Li}, \mathrm{t}\right)^{17} \mathrm{O}$ reaction obtained at 28 and $34 \mathrm{MeV}$, compared with finite-range DWBA calculations normalized to the data.

The contribution of the $1 / 2^{+}$state to the astrophysical S-factor when using the deduced $\gamma_{\alpha}^{2}$ is shown in red curve in Fig. 7 At the energy of astrophysical interest, $E_{c m}=0.19 \mathrm{MeV}$, the contribution of this sub-threshold state to the total S-factor was found dominant $(\approx 70 \%)$ [18]. This confirms the dominant character of the sub-threshold state on the cross section of ${ }^{13} \mathrm{C}(\alpha, \mathrm{n}){ }^{16} \mathrm{O}$ at the energies of astrophysical interest.

\subsubsection{Application of the method to the non-resonant component of ${ }^{60} \mathrm{Fe}(n, \gamma)^{61} \mathrm{Fe}$ reaction}

The observation of ${ }^{60} \mathrm{Fe}$ characteristic gamma-ray lines by RHESSI and INTEGRAL spacecrafts $[28,29]$ as well as its observation in deep ocean crusts, sediments, lunar soils [30-32] and in galagtic cosmic rays (CRIS/ACE) [33] have underlined the need for accurate nuclear information concerning the stellar nucleosynthesis of this nuclei. ${ }^{60} \mathrm{Fe}$ is mainly produced in massive stars through the weak s-process component and it is released in the ISM by the sub-sequent core-collapse supernovae explosion [34]. Its production yield in core-collapse supernovae is uncertain due to the large uncertainties surrounding the cross sections of the production and destruction reactions ${ }^{59} \mathrm{Fe}(\mathrm{n}, \gamma){ }^{60} \mathrm{Fe}$ and ${ }^{60} \mathrm{Fe}(\mathrm{n}, \gamma){ }^{61} \mathrm{Fe}$ respectively.

${ }^{60} \mathrm{Fe}(\mathrm{n}, \gamma){ }^{61} \mathrm{Fe}$ neutron capture reaction proceeds via two mechanisms, a resonant capture above the neutron threshold at $5.58 \mathrm{MeV}$ and a direct capture on the bound states of ${ }^{61} \mathrm{Fe}$ below the threshold. 


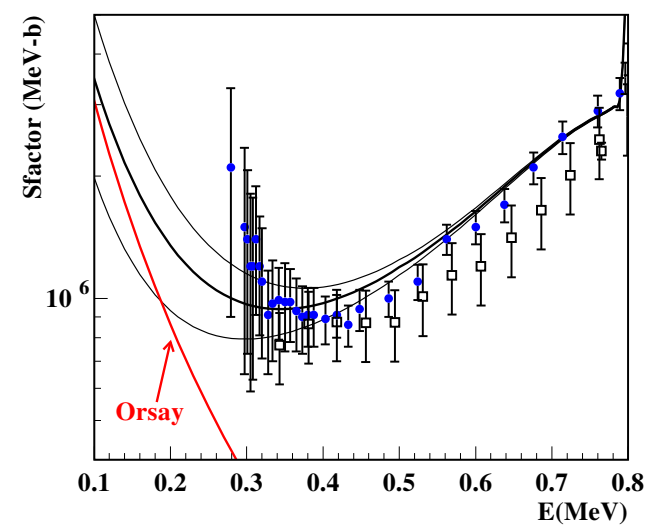

Figure 7. Astrophysical $S$-factor for the ${ }^{13} \mathrm{C}(\alpha, \mathrm{n}){ }^{16} \mathrm{O}$ reaction with $R$-matrix calculations. The data points are taken from Refs [21, 27]. The contribution of the $6.356 \mathrm{MeV}$ state is shown as red curve. The thick black curve corresponds to the recommended $\gamma_{\alpha}^{2}$ values, and the thin black ones to the lower and upper limits.

To evaluate the reaction rate, Hauser Feschbach calculations are used to estimate the resonant capture component while the direct component is determined using the equations described in section 2.2 and the spectroscopic informations $\left(\mathrm{E}_{x}, 1, \mathrm{C}^{2} \mathrm{~S}\right)$ of ${ }^{61} \mathrm{Fe}$ provided by shell model calculations since none of the measured states have known neutron spectroscopic factors.

As mentionned earlier to evaluate the direct component of an (n, $\gamma)$ cross-section, one needs to determine the $C^{2} S$, Ex and 1 of the final bound states and (d,p) transfer reaction is the best tool to determine experimentally these parameters. $\mathrm{An}^{60} \mathrm{Fe}(\mathrm{d}, \mathrm{p} \gamma){ }^{61} \mathrm{Fe}$ experiment was performed at GANIL in inverse kinematics [35] in order to check the reliability of the shell model calculations in the mass region $\mathrm{N}=34$ and evaluate the contribution of the direct component to the total neutron capture cross section. An ${ }^{60} \mathrm{Fe}$ secondary beam was produced by fragmentation of ${ }^{64} \mathrm{Ni}$ on Be target at LISE spectrometer and then sent on a $\mathrm{CD}_{2}$ target. Two MWPC called CATS were used to track the beam for proton emission point localisation. The energy and the emission angle of the emitted protons were detected at backward angles by four MUST2 telescopes and the annular detector S1 from micron. The emitted $\gamma$-rays from the decay of the populated states in ${ }^{61} \mathrm{Fe}$ were detected in 4 EXOGAM clovers. The aim of detecting the $\gamma$-rays is to disentangle the different populated states in ${ }^{61} \mathrm{Fe}$ that can not be discriminate with particle detection. Finally the quasi-projectiles were identified in mass and charge using the ToF and energy loss of the fragments in the ionsation chambre followed by a plastic scintillator.

The obtained ${ }^{61} \mathrm{Fe}$ excitation energy spectrum, using the MUST2 Silicon telescopes, is displayed in figure Fig. 8. Two peaks are observed below the neutron threshold and their width was found much larger than the expected energy resolution of about $800 \mathrm{keV}$. This is an indication of a population of two or more states in the observed peaks that the $\gamma$-rays measurements may help to disentangle.

From the comparison of the excitation energy spectrum with and without $\gamma$-ray coincidence and from the observation of the gamma-ray spectra corresponding to two energy gates in the first peak [35], 0 to $1 \mathrm{MeV}$ and 1 to $2 \mathrm{MeV}$, three states were identified clearly: the known $207 \mathrm{keV}$, the 391 $\mathrm{keV}$ and the isomeric state at $861 \mathrm{keV}$. No other known states above $1 \mathrm{MeV}$ [36] that were maybe 


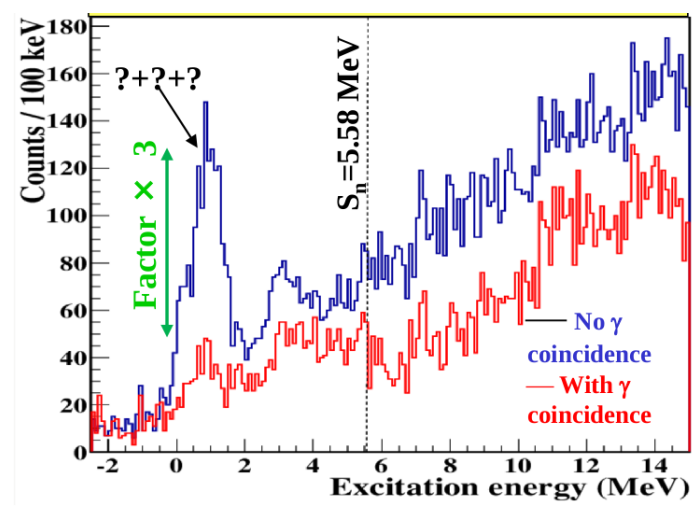

Figure 8. Measured excitation energy spectrum of ${ }^{61} \mathrm{Fe}$ with MUST2 telescopes

populated in this work could be identified reliably because of the large background and the low measured statistics due to the weak population of these states on one hand and the decrease of the $\gamma$-ray detection efficiency at high energies on the other hand.

A deconvolution of the first peak observed in ${ }^{61} \mathrm{Fe}$ excitation energy spectrum was performed with the ground state, the three well identified populated states in this experiment, the $207 \mathrm{keV}\left(\mathrm{J}^{\pi}=5 / 2^{-}\right)$, the $391 \mathrm{keV}\left(\mathrm{J}^{\pi}=1 / 2^{-}\right)$and the $861 \mathrm{keV}\left(\mathrm{J}^{\pi}=9 / 2^{+}\right)$and also a higher level centered at $1600 \mathrm{keV}$ representing a mixture of the non-identified higher states between $1 \mathrm{MeV}$ and $2 \mathrm{MeV}$. Proton angular distributions, displayed in Fig. 9 could be extracted for the $861 \mathrm{keV}$ state and for the excitation energy range corresponding to $207 \mathrm{keV}$ state where an overlap of the ground state and the $391 \mathrm{keV}$ states is present. The red curves are Adiabatic distorded wave approximation (ADWA) calculations performed using Fresco code. A neutron spectroscopic factor of 0.34 was obtained for the f5/2 state from the fit of the data with a combination of an $\mathrm{L}=3$ describing the $207 \mathrm{keV}$ state and an $\mathrm{L}=1$ describing the $\mathrm{p} 1 / 2$ of the $391 \mathrm{keV}$ state and the $\mathrm{p} 3 / 2$ of the $\mathrm{g}$.s for which the spectroscopic factors are fixed to the values 0.58 and 0.15 respectively obtained from the normalisation of the ADWA calculation to the single extracted data point at $4^{\circ}$ from S1 telescope (see [35] for details). For the $861 \mathrm{keV}$ state, a neutron spectrosopic factor of 0.38 was obtained from the normalisation of the calculations to the data.

A comparison between the neutron spectroscopic factors obtained in this work with those predicted in LNPS shell model calculations [37] shows a very good agreement between the experimental results and the calculations. This is an indication of the relaibility of these shell model calculations in the $\mathrm{N}=34$ mass region.

The direct component of ${ }^{60} \mathrm{Fe}(\mathrm{n}, \gamma){ }^{61} \mathrm{Fe}$ was calculated using in TEDCA code [38] the extracted experimental neutron spectroscopic factors for the first four excited states of ${ }^{61} \mathrm{Fe}$. A value of about 0.2 mbarn was obtained at $25 \mathrm{keV}$ which represents only $2 \%$ of the total cross section.

\section{Conclusions}

In both resonant and non resonant capture reactions, the spectroscopic properties $\left(\mathrm{J}^{\pi}, \mathrm{E}_{r}, \mathrm{C}^{2} \mathrm{~S}(\Gamma)\right)$ of the involved states are needed to compute the reaction rates. We have seen that transfer reaction is a good alternative method that can be used to extract these useful spectroscopic informations. However, the optical potential parameters used for the DWBA calculations need to be carefully determined in 

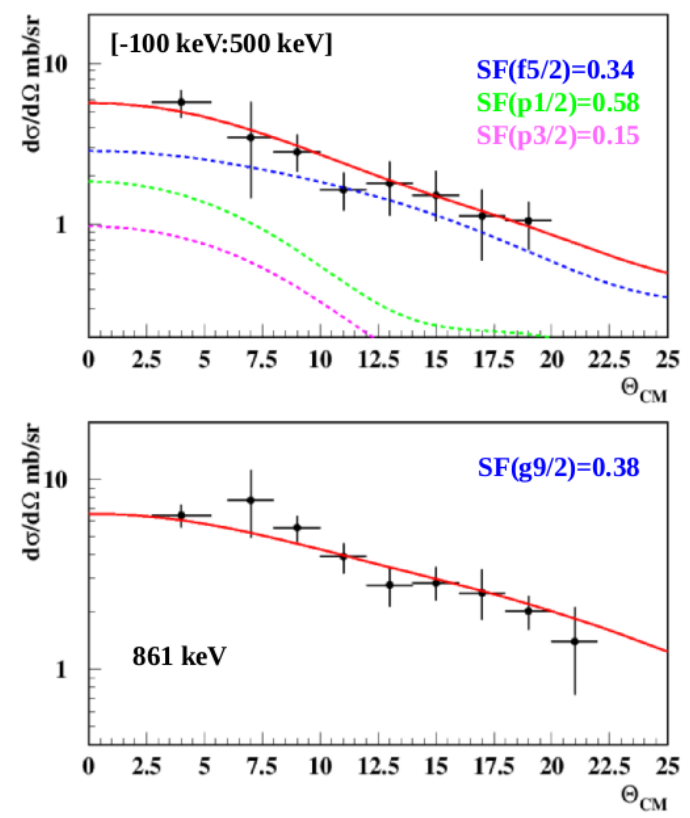

Figure 9. Experimental differential cross sections for a) the excitation energy range corresponding to the 207 $\mathrm{keV}$ state and b) the $861 \mathrm{keV}$ excited state, together with the zero-range ADWA calculations normalized to the data. See text for details.

one hand and in the other hand one should also check the dominant character of the one step direct mechanism in the performed transfer reaction experiment.

\section{References}

[1] R. Satchler, Direct nuclear reaction, Clarendon Press, international series of monograohs on physics, 68 Ed., 1983

[2] G. Baur, C. A. Bertulani, H. Rebel, Nucl. Phys. f 458, 188 (1986).

[3] A. M. Mukhamedzhanov, R.E. Tribble, Phys.Rev.C 59, 3418 (1999).

[4] C. Spitaleri et al., Phys.Rev.C 60, 055802 (1999).

[5] E. Kraussmann et al., Phys. Rev. C 53, 469 (1996)

[6] F. D. Becchetti and J. Jänecke, Nucl. Phys. A 305, 293 (1978)

[7] I.J. Thomson et al., Comp. Phys. Rep. 7, 167 (1988)

[8] P. D. Kunz spot.Colorado.edu/ kunz/DWBA.html

[9] Perey C. M. and Perey F. G. Atom. nucl. Data Tabl. 17 (1976)

[10] W. W. Daehnick et al., Physical Review C 21, 6 (1980)

[11] A. Parikh et al., Phys. Lett. B 737, 314 (2014)

[12] Z. Ma et al. Phys.Rev.C 76, 015803 (2007).

[13] Bardayan et al. Phys.Rev.C 65, 032801 (2002).

[14] G. L. Wales and R. C. Johnson, Nucl. Phys. 274, 168 (1976) 
[15] K. Kemper et al., Aust. J. Phys. 33, 197 (1980)

[16] P. T. Debevec et al., Phys. Rev. C. 9, 2451 (1974)

[17] N. Oulebsir et al., Phys. Rev. C 85035804 (2012) and references therein

[18] M.G. Pellegriti et al., Phys. Rev. C 77042801 (2008) and references therein

[19] R. Talwar et al., Phys.Rev. C 93, 055803 (2016) and references therein

[20] R. Gallino et al., Astrophys. J. 497, 388 (1998)

[21] H. W. Drotleff et al., Astrophys. J. 414, 735 (1993)

[22] P. Descouvemont, Phys. Rev. C. 36, 2206 (1987)

[23] S. Kubono et al., Phys. Rev. lett. 90, 062501 (2003)

[24] N. Keeley et al., Nucl. Phys. A 726, 159 (2003)

[25] P. Schumacher et al., Nucl. Phys. A 212, 573 (1973)

[26] J. D. Garrett et al., Nucl. Phys. A 212, 600 (1973)

[27] C. R. Brune et al. Phys.Rev. C 48, 3119 (1993)

[28] Smith et al., New Astr. ReV. 48, 87 (2004)

[29] W. Wang et al., A and A 469, 1005 (2007)

[30] K. Knie et al., Phys Rev Letter 93, 171103 (2004)

[31] A. Wallner et al., Nature 532, 69 (2016)

[32] L. Fimiani et al., Phys. Rev. Lett. 116, 151104 (2016)

[33] W. R. Binns et al., Science 352, 677 (2016)

[34] M. Limongi and A. Chiefi, New Astronomy Reviews 50, 474 (2006)

[35] S. Giron et al., Phys.Rev. C 95, 035806 (2017)

[36] D. Radulov et al., Phys.Rev. C 88, 014307 (2013)

[37] S.M. Lenzi, F. Nowacki, A. Poves and K. Sieja, Phys. Rev. 82, 054301 (2010)

[38] H. Krauss., Computer code TEDCA, (1995) 